TRANSACTIONS OF THE

AMERICAN MATHEMATICAL SOCIETY

Volume 349, Number 12, December 1997, Pages 4953-4972

S $0002-9947(97) 01875-8$

\title{
LOWER BOUNDS FOR DERIVATIVES OF POLYNOMIALS AND REMEZ TYPE INEQUALITIES
}

\author{
TAMÁS ERDÉLYI AND PAUL NEVAI
}

\begin{abstract}
P. Turán [Über die Ableitung von Polynomen, Comositio Math. 7 (1939), 89-95] proved that if all the zeros of a polynomial $p$ lie in the unit interval $I \stackrel{\text { def }}{=}[-1,1]$, then $\left\|p^{\prime}\right\|_{L^{\infty}(I)} \geq \sqrt{\operatorname{deg}(p)} / 6\|p\|_{L^{\infty}(I)}$. Our goal is to study the feasibility of $\lim _{n \rightarrow \infty}\left\|p_{n}^{\prime}\right\|_{X} /\left\|p_{n}\right\|_{Y}=\infty$ for sequences of polynomials $\left\{p_{n}\right\}_{n \in \mathbb{N}}$ whose zeros satisfy certain conditions, and to obtain lower bounds for derivatives of (generalized) polynomials and Remez type inequalities for generalized polynomials in various spaces.
\end{abstract}

\section{INTRODUCTION}

Markov and Bernstein type inequalities yield estimates of various norms of the derivatives of polynomials in terms of the (possibly different) norms of the polynomials themselves. For instance, the original A. A. Markov (cf. [11, p. 141]) inequality states that ${ }^{1}$

$$
\left\|p^{\prime}\right\|_{L^{\infty}(\Delta)} \leq \frac{2[\operatorname{deg}(p)]^{2}}{|\Delta|}\|p\|_{L^{\infty}(\Delta)}
$$

for every algebraic polynomial $p$ and interval $\Delta \subset \mathbb{R}$. Inequality (1) is sharp; if $n \in \mathbb{N}$ and $p$ is the first kind Chebyshev polynomial of degree $n$ associated with the interval $\Delta$, then (1) turns into an equality. Such inequalities play an essential role in proving inverse type theorems for approximation by polynomials; they are the crucial ingredient of Bernstein's machinery. It didn't take long to realize that (1) is no longer optimal for polynomials satisfying additional conditions. For instance, if $p$ vanishes at the end points of $\Delta$ then according to a (sharp) inequality of I. Schur [15, Theorem IV, p. 282]

$$
\left\|p^{\prime}\right\|_{L^{\infty}(\Delta)} \leq \frac{2[\operatorname{deg}(p)] \cot \frac{\pi}{2 \operatorname{deg}(p)}}{|\Delta|}\|p\|_{L^{\infty}(\Delta)} .
$$

Received by the editors April 20, 1996.

1991 Mathematics Subject Classification. Primary 33A65; Secondary 26C05, 42C05.

Key words and phrases. Markov type inequalities, Remez type inequalities, Turán type inequalities, derivatives, algebraic polynomials, trigonometric polynomials, generalized polynomials.

This material is based upon work supported by the National Science Foundation under Grants No. DMS-9024901 (both authors) and No. DMS-940577 (P. N.).

${ }^{1}$ In what follows, if $A$ is a Lebesgue measurable subset of either $\mathbb{R}$ or $\mathbb{T} \stackrel{\text { def }}{=} \mathbb{R} /[0,2 \pi)$, then the Lebesgue measure of $A$ is denoted by either $m(A)$ or $|A|$.

(C)1997 American Mathematical Society 
Under the even more restrictive condition that all the zeros of $p$ are real and lie outside $\Delta$, P. Erdős [6, Theorem p. 310] and, according to P. Erdős, J. Erőd ${ }^{2}$ proved the (asymptotically sharp) inequality

$$
\left\|p^{\prime}\right\|_{L^{\infty}(\Delta)} \leq \frac{e \operatorname{deg}(p)}{|\Delta|}\|p\|_{L^{\infty}(\Delta)} .
$$

It was their good friend P. Turán [16] who asked the very natural question as to estimating polynomials in terms of their derivatives. He proved that, if all the zeros of $p$ lie in the unit disk $D \stackrel{\text { def }}{=}\{z:|z| \leq 1\}$, then

$$
\left\|p^{\prime}\right\|_{L^{\infty}(D)} \geq \frac{\operatorname{deg}(p)}{2}\|p\|_{L^{\infty}(D)}
$$

[16, Satz I, p. 90], and, if all the zeros of $p$ lie in the unit interval $I \stackrel{\text { def }}{=}[-1,1]$, then

$$
\left\|p^{\prime}\right\|_{L^{\infty}(I)} \geq \frac{\sqrt{\operatorname{deg}(p)}}{6}\|p\|_{L^{\infty}(I)}
$$

[16, Satz II, p. 91]. As a matter of fact, Turán proved that

$$
\left|p^{\prime}(z)\right| \geq \frac{\operatorname{deg}(p)}{2}|p(z)|, \quad z \in \partial D,
$$

as long as all the zeros of $p$ lie in $D$, from which not only (4) follows but also ${ }^{3}$

$$
\left\|p^{\prime}\right\|_{L_{d \mu}^{r}(\partial D)} \geq \frac{\operatorname{deg}(p)}{2}\|p\|_{L_{d \mu}^{r}(\partial D)},
$$

for all $0 \leq r \leq \infty$ and for all nonnegative Borel measures $\mu$ on the unit circle $\partial D$.

The proof of (6) is surprisingly natural and easy, as opposed to the rather technical and counterintuitive proof of (5). Therefore, the analogue of (7) for polynomials with real zeros was and is a much more delicate problem. As a matter of fact, no such inequality is known in weighted $L^{q}$ spaces.

The strongest result to date is due to S. P. Zhou [18, Theorem 4, p. 314], who proved that, if $0<r \leq q \leq \infty$ and $1 \geq 1 / r-1 / q$, then the inverse Markov-Nikol'skiı type inequality

$$
\left\|p^{\prime}\right\|_{L^{r}(I)} \geq \mathrm{C}[\operatorname{deg}(p)]^{\frac{1}{2}-\frac{1}{2 r}+\frac{1}{2 q}}\|p\|_{L^{q}(I)},
$$

holds with a constant $\mathrm{C}=\mathrm{C}(r, q)>0$ for every polynomial $p$ whose zeros lie in the interval $I$. We refer to the references of $[18$, pp. 117-118] for a list of papers on this subject.

Our goal is to study a less ambitious analogue of (7). Namely, in Theorem 2 we investigate the feasibility of

$$
\lim _{n \rightarrow \infty} \frac{\left\|p_{n}^{\prime}\right\|_{X}}{\left\|p_{n}\right\|_{Y}}=\infty
$$

for sequences of polynomials $\left\{p_{n}\right\}_{n \in \mathbb{N}}$ whose zeros satisfy certain conditions.

Our interest in this problem originates from the study of necessary conditions for the convergence of various approximation processes when one desperately needs

\footnotetext{
${ }^{2}$ We thank József Szabados for finding out from Paul Erdős that Erőd never published his result.

${ }^{3}$ Here and in what follows, if $\mu \geq 0$ is a measure or $w \geq 0$ is a function in a set $\Omega$, then $\|\cdot\|_{L_{d \mu}^{p}(\Omega)} \stackrel{\text { def }}{=}\left(\int_{\Omega}|\cdot|^{p} d \mu\right)^{\frac{1}{p}}$ and $\|\cdot\|_{L_{w}^{p}(\Omega)} \stackrel{\text { def }}{=}\left(\int_{\Omega}|\cdot|^{p} w\right)^{\frac{1}{p}}$, respectively. For convenience, we will use these notations for all $0<p<\infty$. If $\mu$ is the Lebesgue measure or $w \equiv 1$, we will simply write $\|\cdot\|_{L^{p}(\Omega)}$.
} 
limit relations such as (9). Luckily, in most applications one has the necessary information about the zeros of the polynomials.

Definition 1. Given a polynomial $p$ and a set $\Omega \subset \mathbb{C}$, the number of zeros of $p$ in $\Omega$, counting multiplicities, is denoted by $Z_{\Omega}(p)$.

Our inverse Markov-Nikol'skii type inequality is the following theorem.

Theorem 2. Let $1 \leq r \leq \infty, 0<q<\infty, \ell \in \mathbb{N}$, and $m \in \mathbb{N}$. Let $\left\{\Delta_{k} \subset \mathbb{R}\right\}_{k=1}^{m}$ be a collection of disjoint bounded intervals of positive length, and let $\Omega$ be a bounded domain in $\mathbb{C}^{+} .{ }^{4}$ Let $\Delta=\bigcup_{k=1}^{m} \Delta_{k}$. Let $u: \Delta \rightarrow \mathbb{R}^{+}, v: \Delta \rightarrow \mathbb{C}$, and $w: \Delta \rightarrow \mathbb{R}^{+}$ be such that $w \in L^{1}(\Delta)$, $u$ is positive on a subset of $\Delta$ with positive Lebesgue measure, $(|v| u)^{q} w \in L^{1}(\Delta), v \neq 0$ on a subset of $\Delta$ with positive Lebesgue measure, and $v^{(\ell-1)}$ is absolutely continuous in every closed subinterval of the interior of each $\Delta_{k}, k=1,2, \ldots, m$. Assume that for each $k=1,2, \ldots, m$, either $u^{-1} \in L^{\infty}\left(\Delta_{k}\right)$ or else the function $u$ is bounded and monotonic in $\Delta_{k}$. Let $\left\{p_{n}\right\}_{n \in \mathbb{N}}$ be a sequence of algebraic polynomials with zeros in $\mathbb{C}^{+}$such that $\lim _{n \rightarrow \infty} Z_{\Omega}\left(p_{n}\right)=\infty$. Then

$$
\lim _{n \rightarrow \infty} \frac{\left\|\left(p_{n} v\right)^{(\ell)} u\right\|_{L^{r}(\Delta)}}{\left\|p_{n} v u\right\|_{L_{w}^{q}(\Delta)}}=\infty .
$$

Remark 3. Without some restrictions on the location of the zeros of $\left\{p_{n}\right\}_{n \in \mathbb{N}}$, formula (10) is not necessarily true. One of the many examples may be given by $p_{n}(x) \stackrel{\text { def }}{=} 1+x^{n}$ with $r=1, u \equiv 1, v \equiv 1$, and $\Delta=[0,1]$. Then all the zeros of $p_{n}$ are in the unit disk, but

$$
\frac{\left\|p_{n}^{\prime}\right\|_{L^{1}(\Delta)}}{\left\|p_{n}\right\|_{L_{w}^{q}(\Delta)}} \leq \frac{1}{\left(\int_{\Delta} w\right)^{\frac{1}{q}}}
$$

Other examples for

$$
\limsup _{n \rightarrow \infty} \frac{\left\|\left(p_{n} v\right)^{(\ell)} u\right\|_{L^{r}(\Delta)}}{\left\|p_{n} v u\right\|_{L_{w}^{q}(\Delta)}}<\infty
$$

may be based on the inequality

$$
\frac{\left|p^{\prime}(x)\right|}{|p(x)|} \leq \frac{\operatorname{deg}(p)}{\operatorname{dist}\{x, \operatorname{zeros}(p)\}},
$$

which follows from the partial fraction decomposition of $p^{\prime} / p$.

Remark 4. In general, the weight $w$ in (10) cannot be replaced by positive Borel measures. For instance, if $\Delta=[a, b], u$ is bounded and nondecreasing on $\Delta, v \equiv 1$, and $d \mu=w d x+J \delta(x-b)$, that is, we have a mass $J$ at $b$, and if $p_{n} \geq 0$ and $p_{n}^{\prime} \geq 0$ in $\Delta$, then

$$
\frac{\left\|p_{n}^{\prime} u\right\|_{L^{1}(\Delta)}}{\left\|p_{n} u\right\|_{L_{d \mu}^{q}(\Delta)}} \leq \frac{1}{J^{\frac{1}{q}}}
$$

Therefore, it may be interesting to find out what class of positive Borel measures could replace $w$ in (10).

\footnotetext{
${ }^{4}$ Here and in what follows, $\mathbb{R}^{+} \stackrel{\text { def }}{=}\{x \in \mathbb{R}: x \geq 0\}, \mathbb{C}^{+} \stackrel{\text { def }}{=}\{z \in \mathbb{Z}: \Re z \geq 0\}$, and $\mathbb{Z}^{+} \stackrel{\text { def }}{=}\{n \in \mathbb{Z}: n \geq 0\} \equiv \mathbb{N} \cup\{0\}$.
} 
Remark 5. Clearly, $(|v| u)^{q} w \in L^{1}(\Delta)$ must hold. In addition, $w \in L^{1}(\Delta)$ cannot be omitted either. To see this, take, for instance, $r=1, q=1, p_{n}(x)=x^{n}, \Delta=[a, b]$ with $|a|<|b|, v(x) \equiv 1, u(x) \equiv(b-x), v \equiv 1$, and $w(x) \equiv(b-x)^{-1}$. Then the limit in the right-hand side of (1) is equal to 1 .

Remark 6. If $0<r<1$ then (10) is no longer valid for every $0<q<\infty$. Take, for instance, $p_{n}(x)=x^{n}, \ell=1$, and $\Delta=[0,1]$, and let $u \equiv 1, v \equiv 1$, and $w \equiv 1$. Then the right-hand side of $(10)$ is finite whenever $1 \leq 1 / r-1 / q$, that is, if $0<r<1$ and $q \geq r /(1-r)$.

Corollary 7. Let $1 \leq r \leq \infty, \ell \in \mathbb{N}$, and $m \in \mathbb{N}$. Let $\left\{\Delta_{k} \subset \mathbb{R}\right\}_{k=1}^{m}$ be a collection of disjoint bounded intervals of positive length, and let $\Omega$ be a bounded interval in $\mathbb{R}$. Let $\Delta=\bigcup_{k=1}^{m} \Delta_{k}$. Let $u(\geq 0) \in L^{r}(\Delta)$ be positive on a subset of $\Delta$ with positive Lebesgue measure. Assume that for each $k=1,2, \ldots, m$, either $u^{-1} \in$ $L^{\infty}\left(\Delta_{k}\right)$ or else the function $u$ is bounded and monotonic in $\Delta_{k}$. Let $\left\{p_{n}\right\}_{n \in \mathbb{N}}$ be a sequence of algebraic polynomials with zeros in $\mathbb{R}$ such that $\lim _{n \rightarrow \infty} Z_{\Omega}\left(p_{n}\right)=\infty$. Let $\left\{g_{n}\right\}_{n \in \mathbb{N}}$ be a sequence of complex valued functions in $\Delta$ such that $g_{n}^{(\ell-1)}$ is absolutely continuous for every $n \in \mathbb{N}$ and

$$
\limsup _{n \in \mathbb{N}} \max _{1 \leq j \leq \ell}\left\|g_{n}^{(j)}\right\|_{L^{\infty}(\Delta)}<\infty \quad \text { and } \quad \limsup _{n \in \mathbb{N}}\left\|g_{n}^{-1}\right\|_{L^{\infty}(\Delta)}<\infty .
$$

Then

$$
\liminf _{n \rightarrow \infty} \frac{\left\|\left(p_{n} g_{n}\right)^{(\ell)} u\right\|_{L^{r}(\Delta)}}{\left\|p_{n}^{(\ell)} g_{n} u\right\|_{L^{r}(\Delta)}} \geq 1 \quad \text { and } \quad \liminf _{n \rightarrow \infty} \frac{\left\|\left(p_{n} g_{n}\right)^{(\ell)} u\right\|_{L^{r}(\Delta)}}{\left\|p_{n}^{(\ell)} u\right\|_{L^{r}(\Delta)}}>0 .
$$

If $\ell=1$, then (12) remains valid even if the conditions that (i) $\Omega$ is a bounded interval in $\mathbb{R}$ and (ii) the zeros of $\left\{p_{n}\right\}_{n \in \mathbb{N}}$ are in $\mathbb{R}$, are replaced by (i) $\Omega$ is a bounded domain in $\mathbb{C}^{+}$and (ii) the zeros of $\left\{p_{n}\right\}_{n \in \mathbb{N}}$ are in $\mathbb{C}^{+}$, respectively.

Remark 8. Note that in Corollary 7 we assume that the zeros of $\left\{p_{n}\right\}_{n \in \mathbb{N}}$ are real (and $\Omega \subset \mathbb{R}$ ) because, in order to prove it, we need to use Theorem 2 not only with $\left\{p_{n}\right\}_{n \in \mathbb{N}}$ but with $\left\{p_{n}^{(j)}\right\}_{n \in \mathbb{N}}$ for $j=1,2, \ldots, \ell-1$ as well. Although, if the zeros of $\left\{p_{n}\right\}_{n \in \mathbb{N}}$ are real, then, by Rolle's Theorem, $\lim _{n \rightarrow \infty} Z_{\Omega}\left(p_{n}\right)=\infty$ guarantees $\lim _{n \rightarrow \infty} Z_{\Omega}\left(p_{n}^{(j)}\right)=\infty$ for $j>0$, still, generally speaking

$$
\lim _{n \rightarrow \infty} Z_{\Omega}\left(p_{n}\right)=\infty \Rightarrow \lim _{n \rightarrow \infty} Z_{\Omega}\left(p_{n}^{\prime}\right)=\infty, \quad \Omega \subset \mathbb{C}
$$

For instance, ${ }^{5}$ if $q_{n}(z) \stackrel{\text { def }}{=} \sum_{k=1}^{n} z^{k} / k !$ and $p_{n}(z) \stackrel{\text { def }}{=} q_{n}\left(a_{n} z\right)$ where $a_{n}$ is the square root of the absolute value of the smallest (in absolute value) zero of $q_{n}^{\prime}$, then, by Hurwitz's Theorem (cf. [17, p. 6]), $\infty$ is the only limit point for the zeros of $\left\{p_{n}^{\prime}\right\}_{n \in \mathbb{N}}$ since $\lim _{n \rightarrow \infty} q_{n}^{\prime}(z)=\exp (z) \neq 0$ for $z \in \mathbb{C}$, whereas

$$
\lim _{n \rightarrow \infty} Z_{\{z:|z| \leq 1\}}\left(p_{n}\right)=\infty
$$

since $\lim _{n \rightarrow \infty} q_{n}(z)=\exp (z)-1$ and the function $\exp (z)-1$ vanishes infinitely many times on the imaginary line.

The trigonometric analogue of Theorem 2 is the following theorem.

${ }^{5}$ We thank Peter Borwein for this example. 
Theorem 9. Let $1 \leq r \leq \infty, 0<q<\infty, \ell \in \mathbb{N}$, and $m \in \mathbb{N}$. Let $\left\{\Delta_{k} \subset \mathbb{T}\right\}_{k=1}^{m}$ be a collection of disjoint bounded intervals of positive length. Let $\Delta=\bigcup_{k=1}^{m} \Delta_{k}$. Let $u: \Delta \rightarrow \mathbb{R}^{+}, v: \Delta \rightarrow \mathbb{C}$, and $w: \Delta \rightarrow \mathbb{R}^{+}$be such that $w \in L^{1}(\Delta), u$ is positive on a subset of $\Delta$ with positive Lebesgue measure, $(|v| u)^{q} w \in L^{1}(\Delta), v \neq 0$ on a subset of $\Delta$ with positive Lebesgue measure, and $v^{(\ell-1)}$ is absolutely continuous in every closed subinterval of the interior of each $\Delta_{k}, k=1,2, \ldots, m$. Assume that for each $k=1,2, \ldots, m$, either $u^{-1} \in L^{\infty}\left(\Delta_{k}\right)$ or else the function $u$ is bounded and monotonic in $\Delta_{k}$. Let $\left\{t_{n}\right\}_{n \in \mathbb{N}}$ be a sequence of trigonometric polynomials with real zeros such that $\lim _{n \rightarrow \infty} Z_{\mathbb{T}}\left(t_{n}\right)=\infty .{ }^{6}$ Then

$$
\lim _{n \rightarrow \infty} \frac{\left\|\left(t_{n} v\right)^{(\ell)} u\right\|_{L^{r}(\Delta)}}{\left\|t_{n} v u\right\|_{L_{w}^{q}(\Delta)}}=\infty .
$$

Remark 10. Note that in Theorem 9, in contrast to Theorem 2, we deal with trigonometric polynomials with real zeros only. It remains to be seen whether Theorem 2 can be extended to trigonometric polynomials with complex zeros.

Definition 11. The set of all polynomials, real polynomials, trigonometric polynomials, and real trigonometric polynomials is denoted by $\mathcal{P}, \mathcal{P}^{r}, \mathcal{T}$, and $\mathcal{T}^{r}$, respectively. Given $n \in \mathbb{Z}^{+}$, the set of all polynomials, real polynomials, trigonometric polynomials, and real trigonometric polynomials of degree at most $n$ is denoted by $\mathcal{P}_{n}, \mathcal{P}_{n}^{r}, \mathcal{T}_{n}$, and $\mathcal{T}_{n}^{r}$, respectively.

Definition 12. The function

$$
f(z)=\omega \prod_{j=1}^{k}\left|z-z_{j}\right|^{r_{j}}, \quad \omega>0, z_{j} \in \mathbb{C}, z \in \mathbb{C}, r_{j}>0,
$$

is called a nonnegative generalized complex algebraic polynomial, that is, $f \in \mathrm{NGAP}$, of degree $N=\sum_{j=1}^{k} r_{j}$, that is, $f \in \operatorname{NGAP}_{N}$. Similarly, the function

$$
f(z)=\omega \prod_{j=1}^{k}\left|\sin \frac{z-z_{j}}{2}\right|^{r_{j}}, \quad \omega>0, z_{j} \in \mathbb{C}, z \in \mathbb{C}, r_{j}>0,
$$

is called a nonnegative generalized complex trigonometric polynomial, that is, $f \in$ NGTP, of degree $N=\frac{1}{2} \sum_{j=1}^{k} r_{j}$, that is, $f \in \operatorname{NGTP}_{N}$. If $f \in \mathrm{NGAP}$ or $f \in \mathrm{NGTP}$ and $z_{0} \in \mathbb{R}$ is a zero of $f$, then the greatest $r \in \mathbb{R}$ such that $f(\cdot) /\left|\cdot-z_{0}\right|^{r} \in$ NGAP or $f(\cdot) /\left|\sin \left(\left(\cdot-z_{0}\right) / 2\right)\right|^{r} \in \mathrm{NGTP}$, respectively, is called the multiplicity of $z_{0}$.

Remark 13. In what follows we will consider both types of generalized polynomials for real arguments only, and then we can assume without loss of generality that each zero $z_{j}$ appears in conjugate pairs. For instance, in the algebraic case, just write

$$
f(x)=\omega \prod_{j=1}^{k}\left|x-z_{j}\right|^{r_{j} / 2}\left|x-\overline{z_{j}}\right|^{r_{j} / 2}, \quad x \in \mathbb{R},
$$

and in the trigonometric case, write

$$
f(x)=\omega \prod_{j=1}^{k}\left|\sin \frac{x-z_{j}}{2}\right|^{r_{j}}\left|\sin \frac{x-\overline{z_{j}}}{2}\right|^{r_{j}}, \quad x \in \mathbb{T} .
$$

\footnotetext{
${ }^{6}$ In other words, $\lim _{n \rightarrow \infty} \operatorname{deg}\left(t_{n}\right)=\infty$.
} 
For real arguments both types of generalized polynomials have derivatives everywhere except at their real zeros with multiplicities at most 1 . If $f \in \mathrm{NGAP}$ or $f \in \mathrm{NGTP}$ and $z_{0} \in \mathbb{R}$ is a zero of $f$ with multiplicity at most 1 , then both the (finite or infinite) left and right derivatives $f_{ \pm}^{\prime}$ of $f$ are well defined at $z_{0}$ and they have the same absolute values, and in this case we set $\left|f^{\prime}\right| \stackrel{\text { def }}{=}\left|f_{+}^{\prime}\right|$. Therefore, if $f \in \mathrm{NGAP}$ or $f \in \mathrm{NGTP}$ then the (finite or infinite) $\left|f^{\prime}\right|$ is well defined in $\mathbb{R}$.

The following two theorems with $L^{\infty}$ and $L^{p}$ Remez type inequalities for generalized polynomials are quite useful.

Theorem 14. There is an absolute constant $\mathrm{c} \in \mathbb{R}^{+}$such that

$$
\|f\|_{L^{\infty}(\mathbb{T})} \leq \begin{cases}\exp (\operatorname{cdeg}(f) s)\|f\|_{L^{\infty}(\mathbb{T} \backslash A)}, & 0<s<\pi / 2, \\ \exp (\operatorname{cdeg}(f)|\log (2 \pi-s)|)\|f\|_{L^{\infty}(\mathbb{T} \backslash A)}, & \pi / 2 \leq s<2 \pi,\end{cases}
$$

holds for every $f \in \mathrm{NGTP}$ and $A \subset \mathbb{T}$ with $m(A) \leq s$. In particular, given $0<\epsilon<2 \pi$, there exists a constant $\mathrm{c}_{\epsilon} \in \mathbb{R}^{+}$such that if $m(A) \leq 2 \pi-\epsilon$ then the inequality

$$
\|f\|_{L^{\infty}(\mathbb{T})} \leq \exp \left(\mathrm{c}_{\epsilon} \operatorname{deg}(f) m(A)\right)\|f\|_{L^{\infty}(\mathbb{T} \backslash A)}
$$

holds for every $f \in \mathrm{NGTP}$. In other words,

$$
\|f\|_{L^{\infty}(\mathbb{T})} \leq \exp \left(\mathrm{c}_{\epsilon} \operatorname{deg}(f) m(x \in \mathbb{T}: f(x) \geq 1)\right)
$$

holds for every $f \in \mathrm{NGTP}$ as long as the measure of the set $\{x \in \mathbb{T}: f(x) \geq 1\}$ is less than or equal to $2 \pi-\epsilon$.

Remark 15. The case $0<s<\pi / 2$ in (14) was proved in [4, Theorem 2, p. 257], and the case $\pi / 2 \leq s<2 \pi$ in (14) was announced in [3, formula (3.10), p. 174].

Theorem 16. Let $\chi$ be a nonnegative and nondecreasing function in $\mathbb{R}^{+}$such that $\chi(\cdot) /(\cdot)$ is nonincreasing. Let $0<p<\infty$. Given $0<\delta<2 \pi$, there exist two constants $\mathrm{d}_{\delta} \in \mathbb{R}^{+}$and $\mathrm{D}_{\delta} \in \mathbb{R}^{+}$such that the inequality

$$
\int_{\mathbb{T}} \chi^{p}(f) \leq\left(1+\mathrm{D}_{\delta} \exp \left(\mathrm{d}_{\delta} p \operatorname{deg}(f) m(A)\right)\right) \int_{\mathbb{T} \backslash A} \chi^{p}(f)
$$

holds for every $f \in \mathrm{NGTP}$ and for every $A \subset \mathbb{T}$ with $m(A) \leq 2 \pi-\delta$.

Remark 17. This theorem is a slight extension of [5, Theorem 8, p. 247], where it was proved for $7 \pi / 4<\delta<2 \pi$.

Generalized polynomials can be estimated in terms of their derivatives by the following

Theorem 18. Let $1 \leq p<\infty, 0<N<\infty, 0 \leq \Gamma<\infty, 0<\delta<2 \pi, f \in \operatorname{NGTP}_{N}$, and $u \in \mathrm{NGTP}_{\Gamma}$. Assume that $f$ has at least one real zero such that $u$ does not vanish at that zero. Let $\Delta=\bigcup \Delta_{k}$ where (i) the $\Delta_{k} \subseteq \mathbb{T}$ are disjoint intervals, (ii) the closure of each $\Delta_{k}$ contains at least one zero of $f$, (iii) $\delta<m(\Delta) \leq 2 \pi$, and (iv)

$$
\Gamma \max _{k}\left|\Delta_{k}\right| \sup _{\substack{x \in \Delta \\ u(\theta)=0}}|\cot (x-\theta)| \leq \frac{1}{2} .
$$


Then there exist two constants $\mathrm{d}_{\delta} \in \mathbb{R}^{+}$and $\mathrm{D}_{\delta} \in \mathbb{R}^{+}$such that

$$
\|f\|_{L_{u}^{p}(\mathbb{T})} \leq 2 p \max _{k}\left|\Delta_{k}\right|\left(1+\mathrm{D}_{\delta} \exp \left(\mathrm{d}_{\delta} p(N+\Gamma)(2 \pi-m(\Delta))\right)\right)\left\|f^{\prime}\right\|_{L_{u}^{p}(\mathbb{T})} .
$$

The following two corollaries show that the weighted $L^{p}$ type Bernstein and Markov inequalities (see, for instance, [12, Theorems 6.16 and 6.19, pp. 163-164], [13, Theorem 5, p. 242], and [2, Theorem 8.4.7, p. 107]) are sharp for polynomials with sufficiently uniformly distributed zeros.

Corollary 19. Let $1 \leq p<\infty, u \in \mathrm{NGTP}, m \in \mathbb{N}$, and $\ell \in \mathbb{N}$. Let $\left\{t_{n}\right\}_{n \in \mathbb{N}}$ be a sequence of real trigonometric polynomials with $\operatorname{deg}\left(t_{n}\right) \leq m n$ for each $n \in \mathbb{N}$. Assume that there is a constant $\mathrm{d} \in \mathbb{R}^{+}$such that for each $n \in \mathbb{N}$ there is a partition $\bigcup_{i} \tau_{\text {in }}=\mathbb{T}$ where (i) $\tau_{\text {in }} \subset \mathbb{T}$ are disjoint intervals, (ii) the closure of each $\tau_{\text {in }}$ contains at least one zero of $t_{n}$, and (iii) $m\left(\tau_{i n}\right) \leq d / n$. Then there exists a constant $\mathrm{C} \in \mathbb{R}^{+}$independent of $\left\{t_{n}\right\}_{n \in \mathbb{N}}$ such that

$$
\limsup _{n \rightarrow \infty} \frac{n^{\ell}\left\|t_{n}^{(j)}\right\|_{L_{u}^{p}(\mathbb{T})}}{n^{j}\left\|t_{n}^{(\ell)}\right\|_{L_{u}^{p}(\mathbb{T})}} \leq \mathrm{C}, \quad j=0,1, \ldots, \ell-1 .
$$

Corollary 20. Let $\Delta \subset \mathbb{R}$ be an interval with endpoints, say, $a<b$, and let $w_{\Delta}(x) \stackrel{\text { def }}{=} \sqrt{(b-x)(x-a)}$ for $x \in \Delta$. Let $1 \leq p<\infty, u \in \mathrm{NGAP}, m \in \mathbb{N}$, and $\ell \in \mathbb{N}$. Let $\left\{p_{n}\right\}_{n \in \mathbb{N}}$ be a sequence of real algebraic polynomials with $\operatorname{deg}\left(p_{n}\right) \leq m n$ for each $n \in \mathbb{N}$. Assume that there is a constant $\mathrm{d} \in \mathbb{R}^{+}$such that for each $n \in \mathbb{N}$ there is a partition $\bigcup_{i} \tau_{\text {in }}=[0, \pi]$ where (i) $\tau_{\text {in }} \subset[0, \pi]$ are disjoint intervals, (ii) the closure of each $\frac{b-a}{2} \cos \left(\tau_{i n}\right)+\frac{b+a}{2}$ contains at least one zero of $p_{n}$, and (iii) $m\left(\tau_{\text {in }}\right) \leq d / n$. Then there exists a constant $\mathrm{C} \in \mathbb{R}^{+}$independent of $\left\{p_{n}\right\}_{n \in \mathbb{N}}$ such that

$$
\limsup _{n \rightarrow \infty} \frac{n^{\ell}\left\|p_{n}^{(j)}\right\|_{L_{u}^{p}(\Delta)}}{n^{j}\left\|w_{\Delta}^{\ell-j} p_{n}^{(\ell)}\right\|_{L_{u}^{p}(\Delta)}} \leq \mathrm{C}, \quad j=0,1, \ldots, \ell-1 .
$$

In Theorem 18 and Corollaries 19 and 20 we apply Remez type inequalities to generalized polynomials to obtain estimates in the entire set $\mathbb{T}$ for trigonometric polynomials and in an interval, say $\Delta$, for algebraic polynomials. In the following theorem and in two of its corollaries we prove similar inequalities for more general functions with sufficiently many zeros which are valid only on subsets of $\mathbb{T}$ or $\Delta$, though these subsets, generally speaking, are close to $\mathbb{T}$ or $\Delta$, and so they are useful in applications.

Theorem 21. Let

$$
u(z) \stackrel{\text { def }}{=} \prod_{j=1}^{k}\left|\sin \frac{z-z_{j}}{2}\right|^{\Gamma_{j}}, \quad z_{j} \in \mathbb{T}, z \in \mathbb{T}, \Gamma_{j} \in \mathbb{R}, 2 \Gamma \stackrel{\text { def }}{=} \sum_{j}\left|\Gamma_{j}\right|,
$$

be fixed. Let $\tau=\bigcup_{i} \tau_{i}$ where $\tau_{i} \subset \mathbb{T}$ are disjoint intervals. Let $1 \leq p<\infty$ and let $\epsilon>0$. Let $f$ be differentiable almost everywhere in each $\tau_{i}$, and let $|f|^{p}$ be absolutely continuous in each $\tau_{i}$. Let the closure of each $\tau_{i}$ contain at least one zero of $f$. Let $\tau_{i_{k}}$ be those intervals $\tau_{i_{k}}$ for which $\operatorname{dist}\left(\tau_{i_{k}},\left\{z_{j}\right\}\right) \geq \epsilon$. Let $\Delta=\bigcup_{k} \tau_{i_{k}}$. Then

$$
\|f\|_{L_{u}^{p}(\Delta)} \leq p \sup _{i} m\left(\tau_{i}\right) \exp \left(\sup _{i} m\left(\tau_{i}\right) \Gamma \pi / \epsilon\right)\left\|f^{\prime}\right\|_{L_{u}^{p}(\Delta)} .
$$


Corollary 22. Let $u$ be given by (22). Let $1 \leq p<\infty, \ell \in \mathbb{N}$, and let $\left\{\epsilon_{n}\right\}_{n \in \mathbb{N}}$ be a positive sequence such that $\liminf _{n \rightarrow \infty} n \epsilon_{n}>0$. Let $\left\{f_{n}\right\}_{n \in \mathbb{N}}$ be such that $f_{n}^{(\ell)}$ exists almost everywhere in $\mathbb{T}$, and $\left|f_{n}^{(\ell-1)}\right|^{p}$ is absolutely continuous in $\mathbb{T}$ for each $n \in \mathbb{N}$. If $\ell>1$ then we also assume that each $f_{n}$ is real valued. Assume that there is a constant $\mathrm{d} \in \mathbb{R}^{+}$such that for each $n \in \mathbb{N}$ there is a partition $\bigcup_{i} \tau_{\text {in }}=\mathbb{T}$ where (i) $\tau_{\text {in }} \subset \mathbb{T}$ are disjoint intervals, (ii) the closure of each $\tau_{\text {in }}$ contains at least one zero of $f_{n}$, and (iii) $m\left(\tau_{i n}\right) \leq d / n$. Then there exist a sequence of open sets $\left\{\Delta_{n} \subset \mathbb{T}\right\}_{n \in \mathbb{N}}$ with $\lim _{n \rightarrow \infty} m\left(\mathbb{T} \backslash \Delta_{n}\right)=0$ and a constant $\mathrm{C} \in \mathbb{R}^{+}$independent of $\left\{f_{n}\right\}_{n \in \mathbb{N}}$ such that

$$
\limsup _{n \rightarrow \infty} \frac{n^{\ell}\left\|f_{n}^{(j)}\right\|_{L_{u}^{p}\left(\Delta_{n}\right)}}{n^{j}\left\|f_{n}^{(\ell)}\right\|_{L_{u}^{p}\left(\Delta_{n}\right)}}<\mathrm{C}, \quad j=0,1, \ldots, \ell-1 .
$$

Corollary 23. Let $\Delta \subset \mathbb{R}$ be an interval with endpoints, say, $a<b$, and let $w_{\Delta}(x) \stackrel{\text { def }}{=} \sqrt{(b-x)(x-a)}$ for $x \in \Delta$. Let

$$
u(z) \stackrel{\text { def }}{=} \prod_{j=1}^{k}\left|z-z_{j}\right|^{\Gamma_{j}}, \quad z_{j} \in \Delta, z \in \Delta, \Gamma_{j} \in \mathbb{R},
$$

be fixed. Let $1 \leq p<\infty, \ell \in \mathbb{N}$, and let $\left\{\epsilon_{n}\right\}_{n \in \mathbb{N}}$ be a positive sequence such that $\liminf _{n \rightarrow \infty} n \epsilon_{n}>0$. Let $\left\{f_{n}\right\}_{n \in \mathbb{N}}$ be such that $f_{n}^{(\ell)}$ exists almost everywhere in $\Delta$, and $\left|f_{n}^{(\ell-1)}\right|^{p}$ is absolutely continuous in $\Delta$ for each $n \in \mathbb{N}$. If $\ell>1$ then we also assume that each $f_{n}$ is real valued. Assume that there is a constant $\mathrm{d} \in \mathbb{R}^{+}$ such that for each $n \in \mathbb{N}$ there is a partition $\bigcup_{i} \tau_{\text {in }}=[0, \pi]$ where (i) $\tau_{\text {in }} \subset[0, \pi]$ are disjoint intervals, (ii) the closure of each $\frac{b-a}{2} \cos \left(\tau_{i n}\right)+\frac{b+a}{2}$ contains at least one zero of $p_{n}$, and (iii) $m\left(\tau_{i n}\right) \leq d / n$. Then there exist a sequence of open sets $\left\{\Delta_{n} \subset \Delta\right\}_{n \in \mathbb{N}}$ with $\lim _{n \rightarrow \infty} m\left(\Delta \backslash \Delta_{n}\right)=0$ and a constant $\mathrm{C} \in \mathbb{R}^{+}$independent of $\left\{f_{n}\right\}_{n \in \mathbb{N}}$ such that

$$
\limsup _{n \rightarrow \infty} \frac{n^{\ell}\left\|f_{n}^{(j)}\right\|_{L_{u}^{p}\left(\Delta_{n}\right)}}{n^{j}\left\|w_{\Delta}^{\ell-j} f_{n}^{(\ell)}\right\|_{L_{u}^{p}\left(\Delta_{n}\right)}}<\mathrm{C}, \quad j=0,1, \ldots, \ell-1 .
$$

\section{Proofs of Theorem 2, Corollary 7 , and Theorem 9}

To prove Theorem 2, we need the following lemma.

Lemma 24. Given a number $0<p \leq \infty$, an interval $\Delta \subset \mathbb{R}$ of positive length, a function $\sigma \geq 0$ with $0<\int_{\Delta} \sigma<\infty$, and an integer $k \geq 0$, then there exists a constant $\mathrm{B} \in \mathbb{R}^{+}$such that if $f: \Delta \rightarrow \mathbb{R}$ is $k$-times continuously differentiable in the interior of $\Delta$, then ${ }^{7}$

$$
\inf _{x \in \Delta}\left|f^{(k)}(x)\right| \leq \mathrm{B}\|f\|_{L_{\sigma}^{p}(\Delta)} .
$$

Remark 25. It would be interesting to find the optimal value of the constant B in (27) for various classes of functions. For instance, if $\sigma \equiv 1$, then it is easy to show that the optimal value $B_{\text {opt }}$ of the constant $B$ satisfies

$$
\mathrm{B}_{\mathrm{opt}} \leq(k+1) !\left(\frac{2 k+1}{|\Delta|}\right)^{k+\frac{1}{p}}\|f\|_{L^{p}(\Delta)} .
$$

\footnotetext{
${ }^{7}$ We emphasize that B is independent of $f$.
} 
Remark 26. If $k>0$, then inequality (27) no longer holds for all complex valued functions. To see this, take, for instance, $f \stackrel{\text { def }}{=} \exp (i \lambda \cdot)$.

Proof of Lemma 24. If $k=0$ then for $0<p<\infty$ inequality (27) follows from the (first) mean value theorem for integrals, whereas for $p=\infty$ it is straightforward. Otherwise, we find $k+1$ disjoint closed intervals $\tau_{j} \subset \Delta^{\circ}$ such that $0<\int_{\tau_{j}} \sigma<\infty$ for $j=1,2, \ldots, k+1$. By the case $k=0$, we can pick $k+1$ points $x_{j} \in \tau_{j}$ such that

$$
\left|f\left(x_{j}\right)\right| \stackrel{\text { def }}{=} \min _{x \in \tau_{j}}|f(x)| \leq \mathrm{B}_{1, j}\|f\|_{L_{\sigma}^{p}\left(\tau_{j}\right)}, \quad j=1,2, \ldots, k+1,
$$

$\left(\mathrm{B}_{1, j} \in \mathbb{R}^{+}\right.$is independent of $\left.f\right)$ so that the divided difference

$$
\left[x_{1}, x_{2}, \ldots, x_{k+1} ; f\right] \stackrel{\text { def }}{=} \sum_{j=1}^{k+1} \frac{f\left(x_{j}\right)}{\prod_{k \neq j}\left(x_{j}-x_{k}\right)}
$$

(cf. $[10$, formula (1), p. 7]) satisfies

$$
\left|\left[x_{1}, x_{2}, \ldots, x_{k+1} ; f\right]\right| \leq \mathrm{B}_{2}\|f\|_{L_{\sigma}^{p}(\Delta)}
$$

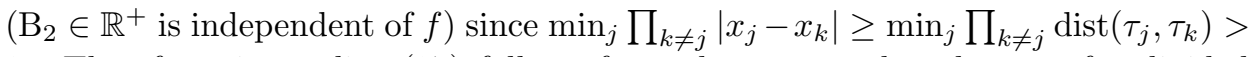
0 . Therefore, inequality (27) follows from the mean value theorem for divided differences (cf. [10, formula (2), p. 6]).

Proof of Theorem 2. The basic idea of the proof is to show that if (10) were not true then there would be a sequence of algebraic polynomials which would converge to an entire function locally uniformly in $\mathbb{C}$ such that (i) this function would not vanish identically in $\mathbb{C}$, and (ii) it would have infinitely many zeros in a bounded domain. The details are given as follows.

In view of Hölder's inequality, it is sufficient to prove (10) for $r=1$.

Step 1. We will prove (10 for $m=1\left(\Delta=\Delta_{1}\right)$ when $u \equiv 1$. We can assume that $|v|^{q} w>0$ on a set of positive Lebesgue measure.

If (10) were not true then there would be a sequence of algebraic polynomials $\left\{\tilde{p}_{n}\right\}_{n \in \mathbb{N}}$ with zeros in $\mathbb{C}^{+}$satisfying

$$
\begin{gathered}
\text { (i) } \lim _{n \rightarrow \infty} Z_{\Omega}\left(\tilde{p}_{n}\right)=\infty, \quad \text { (ii) }\left\|\tilde{p}_{n} v\right\|_{L_{w}^{q}(\Delta)}=1, \\
\text { (iii) } \sup _{n \in \mathbb{N}}\left\|\left(\tilde{p}_{n} v\right)^{(\ell)}\right\|_{L^{1}(\Delta)}<\infty .
\end{gathered}
$$

We claim that the sequences $\left\{\tilde{p}_{n} v\right\}_{n \in \mathbb{N}}$ and $\left\{\left(\tilde{p}_{n} v\right)^{\prime}\right\}_{n \in \mathbb{N}}$ are bounded in $L^{\infty}(\Delta)$ and $L^{1}(\Delta)$, respectively, that is,

$$
\sup _{n \in \mathbb{N}}\left\|\tilde{p}_{n} v\right\|_{L^{\infty}(\Delta)}<\infty \quad \text { and } \quad \sup _{n \in \mathbb{N}}\left\|\left(\tilde{p}_{n} v\right)^{\prime}\right\|_{L^{1}(\Delta)}<\infty .
$$

Applying Lemma 24 with $p=q, k=\ell-1, \sigma \equiv w$, and $f \equiv \Re\left(\tilde{p}_{n} v\right)$, and using (ii) and (iii) in (28), we obtain

$$
\sup _{n \in \mathbb{N}}\left\|\Re\left(\tilde{p}_{n} v\right)^{(\ell-1)}\right\|_{L^{\infty}(\Delta)}<\infty,
$$

and, similarly,

$$
\sup _{n \in \mathbb{N}}\left\|\Im\left(\tilde{p}_{n} v\right)^{(\ell-1)}\right\|_{L^{\infty}(\Delta)}<\infty
$$


that is, (29) holds if $\ell=1$. If $\ell>1$, then we can use Lemma 24 repeatedly with $f \equiv \Re\left(\tilde{p}_{n} v\right)$ and $f \equiv \Im\left(\tilde{p}_{n} v\right)$ to show that (30) and (31) together with (ii) in (28) imply

$$
\sup _{n \in \mathbb{N}}\left\|\Re\left(\tilde{p}_{n} v\right)^{(k)}\right\|_{L^{\infty}(\Delta)}<\infty, \quad k=\ell-2, \ell-3, \ldots, 0
$$

and

$$
\sup _{n \in \mathbb{N}}\left\|\Im\left(\tilde{p}_{n} v\right)^{(k)}\right\|_{L^{\infty}(\Delta)}<\infty, \quad k=\ell-2, \ell-3, \ldots, 0,
$$

so that (29) holds for $\ell>0$ as well.

Using (29), we can apply Helly's Selection Theorem (cf. [8, p. 56]) to pick a subsequence $\left\{\tilde{p}_{n_{k}}\right\}_{k \in \mathbb{N}}$ such that $\lim _{n \rightarrow \infty} \tilde{p}_{n_{k}}(x) v(x)$ exists for every $x \in \Delta$. Let $f(x) \stackrel{\text { def }}{=} \lim _{n \rightarrow \infty} \tilde{p}_{n_{k}}(x) 1_{v \neq 0}(x)$ for $x \in \Delta .^{8}$ By Lebesgue's Dominated Convergence Theorem,

$$
1=\left\|\tilde{p}_{n_{k}} v\right\|_{L_{w}^{q}(\Delta)} \underset{k \rightarrow \infty}{\rightarrow}\|f v\|_{L_{w}^{q}(\Delta)},
$$

so that $f$ exists and $f \neq 0$ on a set $E \subset\{t: v(t) \neq 0\} \subset \Delta$ with positive Lebesgue measure. Thus, by a theorem of B. Ya. Levin in [9, Theorem 2, p. 385] (cf. [1, Corollary 1.3 , p. 110]), the limit $f_{c}(z) \stackrel{\text { def }}{=} \lim _{n \rightarrow \infty} \tilde{p}_{n_{k}}(z)$ exists locally uniformly in $\mathbb{C}$, where $f_{c}$ is an entire function (from the Pólya-Obrechkoff class). Since $\lim _{n \rightarrow \infty} Z_{\Omega}\left(\tilde{p}_{n_{k}}\right)=\infty$ (cf. (i) in (28)), by Hurwitz's Theorem (cf. [17, p. 6]), $f_{c} \equiv 0$. This contradicts (32), since $f_{c} v \equiv f v$ in $\Delta$. Therefore,

$$
\lim _{n \rightarrow \infty} \frac{\left\|\left(p_{n} v\right)^{(\ell)}\right\|_{L^{1}(\Delta)}}{\left\|p_{n} v\right\|_{L_{w}^{q}(\Delta)}}=\infty .
$$

Step 2. We will prove (10) for $m=1\left(\Delta=\Delta_{1}\right)$ when $u^{-1} \in L^{\infty}(\Delta)$. This follows immediately from what we have already proved in Step 1 applied with $u^{q} w$ in place of $w$, since

$$
\left\|\left(p_{n} v\right)^{(\ell)} u\right\|_{L^{1}(\Delta)} \geq\left\|u^{-1}\right\|_{L^{\infty}(\Delta)}^{-1}\left\|\left(p_{n} v\right)^{(\ell)}\right\|_{L^{1}(\Delta)} .
$$

Step 3. We will prove (10) for $m=1\left(\Delta=\Delta_{1}\right)$ when $u$ is bounded and monotonic in $\Delta$. We can assume that $u$ does not vanish inside $\Delta$ since otherwise $u$ would vanish on a subinterval of $\Delta$ sharing an endpoint with $\Delta$, and then we could replace $\Delta$ by a smaller interval without changing any of the integrals in (10).

Write $\Delta$ as the union of two intervals, $\Delta=\Delta^{\prime} \cup \Delta^{\prime \prime}$, where $u \in L^{\infty}\left(\Delta^{\prime}\right)$ but $\inf _{\Delta^{\prime}} u=0$ and $u^{-1} \in L^{\infty}\left(\Delta^{\prime \prime}\right)$. If $\left|\Delta^{\prime}\right|=0$ then we are back to Step 2 and, therefore, (10) holds. Otherwise, we can always assume that $\left|\Delta^{\prime \prime}\right|>0$, and then

$$
\begin{aligned}
\frac{\left\|\left(p_{n} v\right)^{(\ell)} u\right\|_{L^{1}(\Delta)}}{\left\|p_{n} v u\right\|_{L_{w}^{q}(\Delta)}} & =\frac{\left\|\left(p_{n} v\right)^{(\ell)} u\right\|_{L^{1}\left(\Delta^{\prime}\right)}+\left\|\left(p_{n} v\right)^{(\ell)} u\right\|_{L^{1}\left(\Delta^{\prime \prime}\right)}}{\left(\left\|p_{n} v u\right\|_{L_{w}^{q}\left(\Delta^{\prime}\right)}^{q}+\left\|p_{n} v u\right\|_{L_{w}^{q}\left(\Delta^{\prime \prime}\right)}^{q}\right)^{\frac{1}{q}}} \\
& \geq \frac{1}{2^{\frac{1}{q}}} \min \left\{\frac{\left\|\left(p_{n} v\right)^{(\ell)} u\right\|_{L^{1}\left(\Delta^{\prime}\right)}}{\left\|p_{n} v u\right\|_{L_{w}^{q}\left(\Delta^{\prime}\right)}}, \frac{\left\|\left(p_{n} v\right)^{(\ell)} u\right\|_{L^{1}\left(\Delta^{\prime \prime}\right)}}{\left\|p_{n} v u\right\|_{L_{w}^{q}\left(\Delta^{\prime \prime}\right)}}\right\} .
\end{aligned}
$$

We need to prove that

$$
\lim _{n \rightarrow \infty} \frac{\left\|\left(p_{n} v\right)^{(\ell)} u\right\|_{L^{1}\left(\Delta^{\prime}\right)}}{\left\|p_{n} v u\right\|_{L_{w}^{q}\left(\Delta^{\prime}\right)}}=\infty
$$

\footnotetext{
${ }^{8}$ Here and in what follows, the characteristic function of a set $\mathcal{E}$ is denoted by $1_{\mathcal{E}}$.
} 
since by Step 2

$$
\lim _{n \rightarrow \infty} \frac{\left\|\left(p_{n} v\right)^{(\ell)} u\right\|_{L^{1}\left(\Delta^{\prime \prime}\right)}}{\left\|p_{n} v u\right\|_{L_{w}^{q}\left(\Delta^{\prime \prime}\right)}}=\infty .
$$

If $(|v| u)^{q} w=0$ a.e. in $\Delta^{\prime}$, then (35) is certainly true. Now assume that $(|v| u)^{q} w>0$ on a set of positive Lebesgue measure in $\Delta^{\prime}$. If (35) were not true then, analogously to Step 1, there would be a sequence of algebraic polynomials $\left\{\tilde{p}_{n}\right\}_{n \in \mathbb{N}}$ with zeros in $\mathbb{C}^{+}$satisfying

$$
\begin{gathered}
\text { (i) } \lim _{n \rightarrow \infty} Z_{\Omega}\left(\tilde{p}_{n}\right)=\infty, \quad \text { (ii) }\left\|\tilde{p}_{n} v u\right\|_{L_{w}^{q}\left(\Delta^{\prime}\right)}=1, \\
\text { (iii) } \sup _{n \in \mathbb{N}}\left\|\left(\tilde{p}_{n} v\right)^{(\ell)} u\right\|_{L^{1}\left(\Delta^{\prime}\right)}<\infty .
\end{gathered}
$$

As in Step 1, we claim that the sequences $\left\{\tilde{p}_{n} v u\right\}_{n \in \mathbb{N}}$ and $\left\{\left(\tilde{p}_{n} v u\right)^{\prime}\right\}_{n \in \mathbb{N}}$ are bounded in $L^{\infty}\left(\Delta^{\prime}\right)$ and $L^{1}\left(\Delta^{\prime}\right)$, respectively, that is,

$$
\sup _{n \in \mathbb{N}}\left\|\tilde{p}_{n} v u\right\|_{L^{\infty}\left(\Delta^{\prime}\right)}<\infty \quad \text { and } \quad \sup _{n \in \mathbb{N}}\left\|\left(\tilde{p}_{n} v\right)^{\prime} u\right\|_{L^{1}\left(\Delta^{\prime}\right)}<\infty .
$$

To this end we pick a closed interval $\Delta^{*} \subset\left(\Delta^{\prime}\right)^{\circ}$ such that $\int_{\Delta^{*}} u^{q} w>0$. We use Lemma 27 in the interval $\Delta^{*}$ with $p=q, k=\ell-1, \sigma \equiv u^{q} w$, and with the functions $f \equiv \Re\left(\tilde{p}_{n} v\right)$ and $f \equiv \Im\left(\tilde{p}_{n} v\right)$, to show that for each $n \in \mathbb{N}$ there are $x_{n} \in \Delta^{*}$ and $y_{n} \in \Delta^{*}$ such that

$$
\sup _{n \in \mathbb{N}}\left|\Re\left(\tilde{p}_{n} v\right)^{(\ell-1)}\left(x_{n}\right)\right| \leq \mathrm{B}^{*}\left\|\tilde{p}_{n} v u\right\|_{L_{w}^{q}\left(\Delta^{*}\right)}
$$

and

$$
\sup _{n \in \mathbb{N}}\left|\Im\left(\tilde{p}_{n} v\right)^{(\ell-1)}\left(y_{n}\right)\right| \leq \mathrm{B}^{*}\left\|\tilde{p}_{n} v u\right\|_{L_{w}^{q}\left(\Delta^{*}\right)}
$$

with a constant $\mathrm{B}^{*}<\infty$, that is, by (ii) in (37),

$$
\sup _{n \in \mathbb{N}}\left|\Re\left(\tilde{p}_{n} v\right)^{(\ell-1)}\left(x_{n}\right)\right| \leq \mathrm{B}^{*} \quad \text { and } \quad \sup _{n \in \mathbb{N}}\left|\Im\left(\tilde{p}_{n} v\right)^{(\ell-1)}\left(y_{n}\right)\right| \leq \mathrm{B}^{*} .
$$

Since

$$
\begin{aligned}
\left|\left(\tilde{p}_{n} v\right)^{(\ell-1)}(x)\right| \leq & \left|\Re\left(\tilde{p}_{n} v\right)^{(\ell-1)}\left(x_{n}\right)\right|+\left|\Im\left(\tilde{p}_{n} v\right)^{(\ell-1)}\left(y_{n}\right)\right| \\
& +\left|\int_{x}^{x_{n}}\right|\left(\tilde{p}_{n} v\right)^{(\ell)}||+\left|\int_{x}^{y_{n}}\right|\left(\tilde{p}_{n} v\right)^{(\ell)}||, \quad x \in \Delta^{\prime}, n \in \mathbb{N},
\end{aligned}
$$

we have

$$
\begin{aligned}
& \left|\left(\tilde{p}_{n} v\right)^{(\ell-1)}(x) u(x)\right| \leq 2 \mathrm{~B}^{*} u(x) \\
& +\frac{u(x)}{\inf _{t \text { between } x \text { and } x_{n}} u(t)}\left|\int_{x}^{x_{n}}\right|\left(\tilde{p}_{n} v\right)^{(\ell)} u||
\end{aligned}
$$

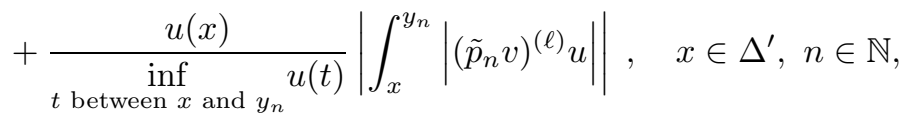

that is,

$$
\begin{aligned}
&\left|\left(\tilde{p}_{n} v\right)^{(\ell-1)}(x) u(x)\right| \leq 2 \mathrm{~B}^{*} u(x) \\
&+2 \sup _{t \in \Delta^{*}}\left(\frac{u(x)}{\min \{u(x), u(t)\}}\right)\left|\int_{x}^{t}\right|\left(\tilde{p}_{n} v\right)^{(\ell)} u||, \quad x \in \Delta^{\prime}, n \in \mathbb{N}
\end{aligned}
$$


( $u$ is monotone between the points $x$ and $x_{n}$ and the points $x$ and $y_{n}$ ), so that

$$
\begin{aligned}
\sup _{n \in \mathbb{N}}\left\|\left(\tilde{p}_{n} v\right)^{(\ell-1)} u\right\|_{L^{\infty}\left(\Delta^{\prime}\right)} & \leq 2 \mathrm{~B}^{*}\|u\|_{L^{\infty}\left(\Delta^{\prime}\right)} \\
& +2\left(1+\frac{\|u\|_{L^{\infty}\left(\Delta^{\prime}\right)}}{\inf _{t \in \Delta^{*}} u(t)}\right) \sup _{n \in \mathbb{N}} \int_{\Delta^{\prime}}\left|\left(\tilde{p}_{n} v\right)^{(\ell)} u\right|<\infty .
\end{aligned}
$$

Therefore, in view of (iii) in (37), the inequalities in (38) hold if $\ell=1$. If $\ell>1$ then we can use the above argument with Lemma 24 repeatedly to show that inequality (ii) in (37), $\sup _{n \in \mathbb{N}}\left\|\left(\tilde{p}_{n} v\right)^{(\ell-1)} u\right\|_{L^{\infty}\left(\Delta^{\prime}\right)}<\infty$, implies

$$
\sup _{n \in \mathbb{N}}\left\|\left(\tilde{p}_{n} v\right)^{(k)} u\right\|_{L^{\infty}\left(\Delta^{\prime}\right)}<\infty, \quad k=\ell-2, \ell-3, \ldots, 0,
$$

and, therefore, (38) holds for $\ell>1$ as well.

Now we pick a sequence of closed intervals $\left\{I_{j}\right\}_{j \in \mathbb{N}}$ such that $I_{j} \subset\left(I_{j+1}\right)^{\circ}$ and $\bigcup_{j \in \mathbb{N}} I_{j}=\left(\Delta^{\prime}\right)^{\circ}$. Then, since $u^{ \pm 1} \in L^{\infty}\left(I_{j}\right)$ for $j \in \mathbb{N}$, by (38),

$$
\sup _{n \in \mathbb{N}}\left\|\tilde{p}_{n} v\right\|_{L^{\infty}\left(I_{j}\right)}<\infty \quad \text { and } \quad \sup _{n \in \mathbb{N}}\left\|\left(\tilde{p}_{n} v\right)^{\prime}\right\|_{L^{1}\left(I_{j}\right)}<\infty, \quad j \in \mathbb{N},
$$

so that, by Helly's Selection Theorem (cf. [8, p. 56]), for each $j \in \mathbb{N}$ we can pick an infinite subsequence $\left\{n_{k, j}\right\}$ such that $\left\{n_{k, j}\right\} \subset\left\{n_{k, j-1}\right\}$, where $\left\{n_{k, 0}\right\} \stackrel{\text { def }}{=} \mathbb{N}$, and $\lim _{n \rightarrow \infty} \tilde{p}_{n_{k, j}}(x) v(x)$ exists for every $x \in I_{j}$. Let $f(x) \stackrel{\text { def }}{=} \lim _{n \rightarrow \infty} \tilde{p}_{n_{k, k}}(x) 1_{v \neq 0}(x)$ for every $x \in\left(\Delta^{\prime}\right)^{\circ}$. Then, by (ii) in (37), (38), and by Lebesgue's Dominated Convergence Theorem,

$$
1=\left\|\tilde{p}_{n_{k, k}} v u\right\|_{L_{w}^{q}\left(\Delta^{\prime}\right)} \underset{k \rightarrow \infty}{\rightarrow}\|f v u\|_{L_{w}^{q}\left(\Delta^{\prime}\right)}
$$

so that $f$ exists and $f \neq 0$ on a set $E \subset \Delta^{\prime} \cap\{t: v(t) \neq 0\}$ with positive Lebesgue measure. Thus, just as in Step 1, by B. Ya. Levin's theorem in [9, Theorem 2, p. 385] (cf. [1, Corollary 1.3, p. 110]), the limit $f_{c}(z) \stackrel{\text { def }}{=} \lim _{n \rightarrow \infty} \tilde{p}_{n_{k, k}}(z)$ exists locally uniformly in $\mathbb{C}$, where $f_{c}$ is an entire function (from the Pólya-Obrechkoff class). Since $\lim _{n \rightarrow \infty} Z_{\Omega}\left(\tilde{p}_{n_{k, k}}\right)=\infty$, by Hurwitz's Theorem (cf. [17, p. 6]), $f \equiv 0$. This contradicts $(39)$ since $f_{c} v \equiv f v$ in $\left(\Delta^{\prime}\right)^{\circ}$. Therefore,

$$
\lim _{n \rightarrow \infty} \frac{\left\|\left(p_{n} v\right)^{(\ell)} u\right\|_{L^{1}(\Delta)}}{\left\|p_{n} v u\right\|_{L_{w}^{q}(\Delta)}}=\infty .
$$

Step 4. The case when $m>1$ follows from the case when $m=1$, since

$$
\begin{aligned}
\frac{\left\|\left(p_{n} v\right)^{(\ell)} u\right\|_{L^{1}(\Delta)}}{\left\|p_{n} v u\right\|_{L_{w}^{q}(\Delta)}(\Delta)} & =\frac{\sum_{k=1}^{m}\left\|\left(p_{n} v\right)^{(\ell)} u\right\|_{L^{1}\left(\Delta_{k}\right)}}{\left(\sum_{k=1}^{m}\left\|p_{n} v u\right\|_{L_{w}^{q}\left(\Delta_{k}\right)}^{q}\right)^{\frac{1}{q}}} \\
& \geq \frac{1}{m^{\frac{1}{q}}} \min _{\substack{1 \leq k \leq m \\
\int_{\Delta_{k}}|v|>0}}\left\{\frac{\left\|\left(p_{n} v\right)^{(\ell)} u\right\|_{L^{1}\left(\Delta_{k}\right)}}{\left\|p_{n} v u\right\|_{L_{w}^{q}\left(\Delta_{k}\right)}}\right\} .
\end{aligned}
$$

Therefore, we have completed the proof of (10) in its entire generality. 
Proof of Corollary 7. By Leibniz's rule, we have

$$
\begin{aligned}
\frac{\left\|\left(p_{n} g_{n}\right)^{(\ell)} u\right\|_{L^{r}(\Delta)}}{\left\|p_{n}^{(\ell)} u\right\|_{L^{r}(\Delta)}\left\|g_{n}^{-1}\right\|_{L^{\infty}(\Delta)}^{-1}} & \geq \frac{\left\|\left(p_{n} g_{n}\right)^{(\ell)} u\right\|_{L^{r}(\Delta)}}{\left\|p_{n}^{(\ell)} g_{n} u\right\|_{L^{r}(\Delta)}} \\
& \geq 1-\sum_{j=1}^{\ell}\left(\begin{array}{l}
\ell \\
j
\end{array}\right) \frac{\left\|p_{n}^{(\ell-j)} u\right\|_{L^{r}(\Delta)}\left\|g_{n}^{(j)}\right\|_{L^{\infty}(\Delta)}}{\left\|p_{n}^{(\ell)} u\right\|_{L^{r}(\Delta)}\left\|g_{n}^{-1}\right\|_{L^{\infty}(\Delta)}^{-1}}
\end{aligned}
$$

so that, in view of (11), Theorem 2, applied to $\left\{p_{n}^{(\ell-j)}\right\}_{n \in \mathbb{N}}$ for $j=1,2, \ldots, \ell$ with $v \equiv 1$ and $w \equiv 1$, immediately yields (12). ${ }^{9}$

Proof of Theorem 9. The proof is essentially the same as that of Theorem 2 with some modifications. Instead of giving a complete proof we will only concentrate on the differences between the two proofs.

In view of Hölder's inequality, it is sufficient to prove (13) for $r=1$. Since the zeros of each $t_{n}$ are real, we can assume that each $t_{n}$ itself is real for $n \in \mathbb{Z}^{+}$as well. Moreover, since

$$
\begin{aligned}
& \frac{\left\|\left(t_{n} v\right)^{(\ell)} u\right\|_{L^{1}(\Delta)}}{\left\|t_{n} v u\right\|_{L_{w}^{q}(\Delta)}} \\
& \quad \geq \frac{1}{2^{\max \{1,1 / q\}}} \min \left\{\frac{\left\|\left(t_{n} \Re v\right)^{(\ell)} u\right\|_{L^{1}(\Delta)}}{\left\|t_{n}(\Re v) u\right\|_{L_{w}^{q}(\Delta)}}, \frac{\left\|\left(t_{n} \Im v\right)^{(\ell)} u\right\|_{L^{1}(\Delta)}}{\left\|t_{n}(\Im v) u\right\|_{L_{w}^{q}(\Delta)}}\right\},
\end{aligned}
$$

we can also assume that $v: \Delta \rightarrow \mathbb{R}$.

Step 1. We will outline the proof of (13) for $m=1\left(\Delta=\Delta_{1}\right)$ when $u \equiv 1$. We can assume that $|v|^{q} w>0$ on a set of positive Lebesgue measure.

If (13) were not true then there would be a sequence of real trigonometric polynomials $\left\{\tilde{t}_{n}\right\}_{n \in \mathbb{N}}$ with real zeros satisfying

$$
\begin{gathered}
\text { (i) } \lim _{n \rightarrow \infty} Z_{\mathbb{T}}\left(\tilde{t}_{n}\right)=\infty, \quad \text { (ii) } \quad\left\|\tilde{t}_{n} v\right\|_{L_{w}^{q}(\Delta)}=1, \\
\text { (iii) } \sup _{n \in \mathbb{N}}\left\|\left(\tilde{t}_{n} v\right)^{(\ell)}\right\|_{L^{1}(\Delta)}<\infty .
\end{gathered}
$$

We claim that

$$
\sup _{n \in \mathbb{N}}\left\|\tilde{t}_{n} v\right\|_{L^{\infty}(\Delta)}<\infty \quad \text { and } \quad \sup _{n \in \mathbb{N}}\left\|\left(\tilde{t}_{n} v\right)^{\prime}\right\|_{L^{1}(\Delta)}<\infty .
$$

Applying Lemma 24 with $p=q, k=\ell-1, \sigma \equiv w$, and $f \equiv \tilde{t}_{n} v$, and using (ii) and (iii) in (41), we obtain

$$
\sup _{n \in \mathbb{N}}\left\|\left(\tilde{t}_{n} v\right)^{(\ell-1)}\right\|_{L^{\infty}(\Delta)}<\infty,
$$

that is, (42) holds if $\ell=1$. If $\ell>1$ then we can use Lemma 24 repeatedly with $f \equiv \tilde{t}_{n} v$ to show that (43) together with (ii) in (41) implies

$$
\sup _{n \in \mathbb{N}}\left\|\left(\tilde{t}_{n} v\right)^{(k)}\right\|_{L^{\infty}(\Delta)}<\infty, \quad k=\ell-2, \ell-3, \ldots, 0,
$$

so that (42) holds for $\ell>0$ as well.

\footnotetext{
${ }^{9}$ Note that if $\ell>1$ then, since the zeros of $\left\{p_{n}\right\}_{n \in \mathbb{N}}$ are real and $\lim _{n \rightarrow \infty} Z_{\Omega}\left(p_{n}\right)=\infty(\Omega \subset \mathbb{R})$, Rolle's Theorem yields $\lim _{n \rightarrow \infty} Z_{\Omega}\left(p_{n}^{(\ell-j)}\right)=\infty$ for $j=1,2, \ldots, \ell-1$, so that Theorem 2 may indeed be used.
} 
Using (42), we can apply Helly's Selection Theorem (cf. [8, p. 56]) to pick a subsequence $\left\{\tilde{t}_{n_{k}}\right\}_{k \in \mathbb{N}}$ such that $\lim _{n \rightarrow \infty} \tilde{t}_{n_{k}}(x) v(x)$ exists for every $x \in \Delta$. Let $f(x) \stackrel{\text { def }}{=} \lim _{n \rightarrow \infty} \tilde{t}_{n_{k}}(x) 1_{v \neq 0}(x)$ for $x \in \Delta$. By Lebesgue's Dominated Convergence Theorem,

$$
1=\left\|\tilde{t}_{n_{k}} v\right\|_{L_{w}^{q}(\Delta)} \underset{k \rightarrow \infty}{\rightarrow}\|f v\|_{L_{w}^{q}(\Delta)},
$$

so that $f$ exists and $f \neq 0$ on a set $E \subset\{t: v(t) \neq 0\} \subset \Delta$ with positive Lebesgue measure. Let $x_{\mathcal{L}} \in E$ be a Lebesgue density point of $E$, that is, let

$$
\lim _{h \downarrow 0} \frac{\left|\left(x_{\mathcal{L}}-h, x_{\mathcal{L}}+h\right) \cap E\right|}{2 h}=1
$$

(cf. [14, Theorem, p. 13]). Fix $0<\epsilon<1$ and pick $0<h<\pi$ such that

$$
\left|\left(x_{\mathcal{L}}-h, x_{\mathcal{L}}+h\right) \cap E\right|>(2-\epsilon) h .
$$

Let $E_{h} \stackrel{\text { def }}{=}\left(E-x_{\mathcal{L}}\right) \cap(-h, h) \cap\left(x_{\mathcal{L}}-E\right) .{ }^{10}$ Then $E_{h} \equiv E_{-h}$ and

$$
\left|E_{h}\right| \geq\left|E-x_{\mathcal{L}}\right|+\left|x_{\mathcal{L}}-E\right|-2 h \geq(2-\epsilon) h+(2-\epsilon) h-2 h=2 h(1-\epsilon)>0,
$$

and, therefore, $\left|\arccos \left(E_{h} \cap(0, h)\right)\right|>0$ as well. Let the sequence of algebraic polynomials $\left\{\tilde{p}_{n_{k}}\right\}_{k \in \mathbb{N}}$ be defined by $\tilde{p}_{n_{k}}(\cos x) \stackrel{\text { def }}{=} t_{n_{k}}\left(x_{\mathcal{L}}+x\right) t_{n_{k}}\left(x_{\mathcal{L}}-x\right)$. Then $\lim _{n \rightarrow \infty} \tilde{p}_{n_{k}}(\cos x)=f\left(x_{\mathcal{L}}+x\right) f\left(x_{\mathcal{L}}-x\right) \neq 0$ for $x \in E_{h} \cap(0, h)$, that is, $f_{r} \stackrel{\text { def }}{=}$ $\lim _{n \rightarrow \infty} \tilde{p}_{n_{k}} \neq 0$ on the set $\arccos \left(E_{h} \cap(0, h)\right) \subset[-1,1]$, which is of positive measure. Thus, by a theorem of B. Ya. Levin in [9, Theorem 2, p. 385] (cf. $\left[1\right.$, Corollary 1.3 , p. 110]), the limit $f_{c}(z) \stackrel{\text { def }}{=} \lim _{n \rightarrow \infty} \tilde{t}_{n_{k}}(z)$ exists locally uniformly in $\mathbb{C}$, where $f_{c}$ is an entire function (from the Pólya-Obrechkoff class). Since $\lim _{n \rightarrow \infty} Z_{[-1,1]}\left(\tilde{p}_{n_{k}}\right)=\infty$ (cf. (i) in (41)), by Hurwitz's Theorem (cf. [17, p. 6]), $f_{c} \equiv 0$. This is a contradiction since $f_{c} \equiv f_{r} \neq 0$ in $\arccos \left(E_{h} \cap(0, h)\right)$. Therefore,

$$
\lim _{n \rightarrow \infty} \frac{\left\|\left(t_{n} v\right)^{(\ell)}\right\|_{L^{1}(\Delta)}}{\left\|t_{n} v\right\|_{L_{w}^{q}(\Delta)}}=\infty .
$$

Step 2. The case when $m=1$ and $u^{-1} \in L^{\infty}(\Delta)$ follows from Step 1 the same way as it does in Step 2 in the proof of Theorem 2 .

Step 3. The case when $m=1$ and $u$ is bounded and monotonic in $\Delta$ is proved analogously to Step 3 in the proof of Theorem 2 using the same modifications as in Step 1, that is, by considering the subsequence

$$
\left\{\tilde{p}_{n_{k}}(\cos \cdot) \stackrel{\text { def }}{=} t_{n_{k}}\left(x_{\mathcal{L}}+\cdot\right) t_{n_{k}}\left(x_{\mathcal{L}}-\cdot\right)\right\}
$$

as opposed to the choice of $\left\{\tilde{p}_{n_{k}}\right\}$ in Step 3 in the proof of Theorem 2.

Step 4. The case when $m>1$ follows from the case when $m=1$ in the same way as it does in Theorem 2 .

Therefore, we have completed the (outline of the) proof of (13) in its entire generality.

\footnotetext{
${ }^{10}$ Here and in what follows, given a set $\Theta$ and numbers $\rho_{1} \in \mathbb{C}$ and $\rho_{2} \in \mathbb{C}$, we use the notation $\rho_{1}+\rho_{2} \Theta \stackrel{\text { def }}{=}\left\{\rho_{1}+\rho_{2} t: t \in \Theta\right\}$.
} 


\section{Proofs of Results on Generalized polynomials}

The proof of Theorem 14 is based on the following lemma.

Lemma 27. There exists a constant $\mathrm{c} \in \mathbb{R}^{+}$such that for every closed interval $\tau \subset \mathbb{T}$ of length $\pi / 4$ and for every $7 \pi / 4 \leq s<2 \pi$ the inequality

$$
\|t\|_{L^{\infty}(\tau-\pi / 4)} \leq \exp (\operatorname{cdeg}(t)|\log (2 \pi-s)|)\|t\|_{L^{\infty}(A)}, \quad t \in \mathcal{T}^{r},
$$

holds for every set $A \subset \tau$ with $m(A) \geq 2 \pi-s$.

Proof of Lemma 27. Without loss of generality we assume $\tau=[\pi / 4, \pi / 2]$. Fix $n \in \mathbb{N}, 7 \pi / 4 \leq s<2 \pi$, and $y \in[0, \pi / 4)$. Let

$$
\mathcal{T}_{n}^{r}(s) \stackrel{\text { def }}{=}\left\{t \in \mathcal{T}_{n}^{r}: m(\{x \in[\pi / 4, \pi / 2]:|t(x)| \leq 1\}) \geq 2 \pi-s\right\} .
$$

A standard compactness argument shows that there exists $t^{*} \in \mathcal{T}_{n}^{r}$ such that

$$
\left|t^{*}(y)\right|=\sup _{t \in \mathcal{T}_{n}^{r}(s)}|t(y)|
$$

We will show that the set

$$
M\left(t^{*}\right) \stackrel{\text { def }}{=}\{x \in[\pi / 4, \pi / 2]:|t(x)| \leq 1\}
$$

is an interval. Clearly, there is $m \in \mathbb{N}$ such that

$$
M\left(t^{*}\right)=\bigcup_{k=1}^{m} M_{k}\left(t^{*}\right),
$$

where the sets $M_{k}\left(t^{*}\right)$ are disjoint closed subintervals of $[\pi / 4, \pi / 2]$. Hence, our job is to show that $m=1$ in (49).

First, we claim that all the zeros of $t^{*}$ are real. If not then $t^{*}(\zeta)=0$ for some $\zeta \in \mathbb{C} \backslash \mathbb{R}$, and then for sufficiently small $\eta_{1}>0$ and $\eta_{2}>0$ the polynomial

$$
\left(1+\eta_{1}\right) t^{*}(z)\left(1-\frac{\eta_{2} \sin ^{2} \frac{z-y}{2}}{\sin \frac{z-\zeta}{2} \sin \frac{z-\bar{\zeta}}{2}}\right)
$$

also belongs to $\mathcal{T}_{n}^{r}(s)$, which contradicts the extremality of $t^{*}$.

Second, we claim that all the zeros of $t^{*}$ (more precisely, those which are in $[0,2 \pi))$ lie in $[\pi / 4, \pi / 2]$. If not then $t^{*}(\zeta)=0$ for some $\zeta \in \mathbb{T} \backslash[\pi / 4, \pi / 2]$, and then for sufficiently small $\eta_{1}>0$ and $\eta_{2}>0$ the polynomial

$$
\left(1+\eta_{1}\right) t^{*}(z)\left(1-\frac{\eta_{2} \operatorname{sgn}\left(\sin \frac{\pi-2 \zeta}{4}\right) \sin \frac{z-y}{2}}{\sin \frac{z-\zeta}{2}}\right)
$$

also belongs to $\mathcal{T}_{n}^{r}(s)$ which contradicts the extremality of $t^{*}$.

Third, we claim that each component $M_{k}\left(t^{*}\right)$ in (49) contains at least one zero of $t^{*}$. This follows from the observation that if there is more then one component, then between consecutive components $t^{*}$ has an extremal point so that $\left(t^{*}\right)^{\prime}$ vanishes there, and, therefore, the the zeros of $t^{*}$ and $\left(t^{*}\right)^{\prime}$ can interlace only if each component contains at least one zero of $t^{*}$.

Finally, we claim that $m$ in (49) is indeed equal to 1 . If not, then let $M_{1}\left(t^{*}\right)$ and $M_{2}\left(t^{*}\right)$ be the closest and the second closest components of $M\left(t^{*}\right)$ to $\pi / 4$, and let 
$\zeta_{j}$ denote the zeros of $t^{*}$ in $M_{1}\left(t^{*}\right)$. Then the polynomial

$$
t^{*}(z) \prod_{j} \frac{\sin \frac{z-\zeta_{j}-\operatorname{dist}\left(M_{1}\left(t^{*}\right), M_{2}\left(t^{*}\right)\right)}{2}}{\sin \frac{z-\zeta_{j}}{2}}
$$

also belongs to $\mathcal{T}_{n}^{r}(s)$, which contradicts the extremality of $t^{*}$.

Hence, $M\left(t^{*}\right)$ in (48) is an interval so that $\left|t^{*}\right| \leq 1$ in an interval of length at least $2 \pi-s$, and then, by [4, Lemma 3, p. 257],

$$
\left\|t^{*}\right\|_{L^{\infty}(\mathbb{T})} \leq T_{2 n}(1 / \sin (\pi / 2-s / 4)),
$$

where $T_{2 n}$ denotes the first kind Chebysev polynomial of degree $2 n$, that is, $T_{2 n}(x)$ $\stackrel{\text { def }}{=} \cos (2 n \arccos x)$. Therefore, by routine estimates,

$$
\left\|t^{*}\right\|_{L^{\infty}(\mathbb{T})} \leq \exp (\mathrm{c} n|\log (2 \pi-s)|),
$$

with an appropriate absolute constant $\mathrm{c}>0$. By the extremality of $t^{*}$ (cf. (47)), we obtain

$$
|t(y)| \leq \exp (\mathrm{c} n|\log (2 \pi-s)|), \quad t \in \mathcal{T}_{n}^{r}(s) .
$$

Finally, pick $t \in \mathcal{T}^{r}$ and $A \subset[\pi / 4, \pi / 2]$ with $m(A) \geq 2 \pi-s$. Let $n=\operatorname{deg}(t)$. Then $t /\|t\|_{L^{\infty}(A)} \in \mathcal{T}_{n}^{r}(s)$, so that (46) in Lemma 27 follows from (50) applied with $t /\|t\|_{L^{\infty}(A)}$ in place of $t$.

Proof of Theorem 14. The case $0<s<\pi / 2$ in (14) was proved in [4, Theorem 2, p. 257], and, therefore, we will consider only the case $\pi / 2 \leq s<2 \pi$ in (14). Let $A \subset \mathbb{T}$ with $m(A) \leq s$. Then there is a closed interval $\tau \subset \mathbb{T}$ such that $m(\tau)=\pi / 4$ and $m((\mathbb{T} \backslash A) \cap \tau) \geq(2 \pi-s) / 8$. By Lemma 27 , applied with $7 \pi / 4+s / 8$ in place of $s$,

$$
\|t\|_{L^{\infty}(\tau-\pi / 4)} \leq \exp (\operatorname{cdeg}(t)|\log (\pi / 4-s / 8)|)\|t\|_{L^{\infty}(\mathbb{T} \backslash A)}, \quad t \in \mathcal{T}^{r} .
$$

On the other hand, by [4, Lemma 3, p. 257],

$$
\|t\|_{L^{\infty}(\mathbb{T})} \leq T_{2 \operatorname{deg}(t)}(1 / \sin (\pi / 16))\|t\|_{L^{\infty}(\tau-\pi / 4)}, \quad t \in \mathcal{T}^{r},
$$

where $T_{n}$ denotes the first kind Chebysev polynomial of degree $n$, that is, $T_{n}(x) \stackrel{\text { def }}{=}$ $\cos (n \arccos x)$. Combining the last two inequalities, we obtain

$$
\begin{aligned}
& \|t\|_{L^{\infty}(\mathbb{T})} \leq T_{2 \operatorname{deg}(t)}(1 / \sin (\pi / 16)) \exp (\operatorname{cdeg}(t)|\log (\pi / 4-s / 8)|)\|t\|_{L^{\infty}(\mathbb{T} \backslash A)}, \\
& t \in \mathcal{T}^{r},
\end{aligned}
$$

that is,

$$
\|t\|_{L^{\infty}(\mathbb{T})} \leq \exp \left(c^{*} \operatorname{deg}(t)|\log (\pi / 4-s / 8)|\right)\|t\|_{L^{\infty}(\mathbb{T} \backslash A)}, \quad t \in \mathcal{T}^{r},
$$

with an appropriate constant $\mathrm{c}^{*} \in \mathbb{R}^{+}$.

If $f \in \mathrm{NGAP}$ is of the form $f(x)=\omega \prod_{j=1}^{k}\left|z-z_{j}\right|^{r_{j}}$ with rational exponents $r_{j}$, say $r_{j}=q_{j} / q$, where $q_{j} \in \mathbb{N}$ and $q \in \mathbb{N}$, then applying (51) with $t \stackrel{\text { def }}{=} f^{2 q}$, the case $\pi / 2 \leq s<2 \pi$ in (14) follows with the same constant $\mathrm{c} \stackrel{\text { def }}{=} \mathrm{c}^{*}$. Once there is an absolute constant $\mathrm{c} \in \mathbb{R}^{+}$such that (14) holds for all $f \in \mathrm{NGAP}$ with rational exponents, it can be extended to all $f \in$ NGAP by approximating the exponents with rational numbers. Therefore, inequality (14) has been completely proved. 
Proof of Theorem 16. We will expand and extend the proof of [5, Theorem 8, p. 251]. Let $0<p<\infty, 0<\delta<2 \pi, f \in \mathrm{NGTP}$, and $A \subset \mathbb{T}$ with $m(A) \leq 2 \pi-\delta$. Let

$$
s=s_{\delta, A} \stackrel{\text { def }}{=} \frac{2 \pi-\delta / 2}{2 \pi-\delta} m(A)
$$

and let $\mathrm{c}_{\delta / 2}$ be defined by (16) applied with $\epsilon=\delta / 2$. Define

$$
I(f) \stackrel{\text { def }}{=}\left\{x \in \mathbb{T}: \chi(f(x)) \geq \exp \left(-c_{\delta / 2} \operatorname{deg}(f) s\right)\|\chi(f)\|_{L^{\infty}(\mathbb{T})}\right\} .
$$

If $f(x) \geq \exp \left(-c_{\delta / 2} \operatorname{deg}(f) s\right)\|f\|_{L^{\infty}(\mathbb{T})}$ then, since $\chi$ is nondecreasing,

$$
\chi(f(x)) \geq \chi\left(\exp \left(-\mathrm{c}_{\delta / 2} \operatorname{deg}(f) s\right)\|f\|_{L^{\infty}(\mathbb{T})}\right),
$$

and then, since $\chi(\cdot) /(\cdot)$ is nonincreasing as well,

$$
\chi(f(x)) \geq \exp \left(-\mathrm{c}_{\delta / 2} \operatorname{deg}(f) s\right) \chi\left(\|f\|_{L^{\infty}(\mathbb{T})}\right) .
$$

Hence $J(f) \subseteq I(f)$, where

$$
J(f) \stackrel{\text { def }}{=}\left\{x \in \mathbb{T}: f(x) \geq \exp \left(-\mathrm{c}_{\delta / 2} \operatorname{deg}(f) s\right)\|f\|_{L^{\infty}(\mathbb{T})}\right\} .
$$

We claim that $m(J(f)) \geq s$. If not, then there is $\eta>0$ such that

$$
m(x \in \mathbb{T}: g(x) \geq 1) \leq s=\frac{2 \pi-\delta / 2}{2 \pi-\delta} m(A) \leq 2 \pi-\delta / 2,
$$

where

$$
g \stackrel{\text { def }}{=}(1+\eta) \exp \left(c_{\delta / 2} \operatorname{deg}(f) s\right)\|f\|_{L^{\infty}(\mathbb{T})}^{-1} f
$$

so that, by (16)

$$
\begin{aligned}
& (1+\eta) \exp \left(\mathrm{c}_{\delta / 2} \operatorname{deg}(f) s\right)=\|g\|_{L^{\infty}(\mathbb{T})} \\
& \quad \leq \exp \left(\mathrm{c}_{\delta / 2} \operatorname{deg}(f) m(x \in \mathbb{T}: g(x) \geq 1)\right) \leq \exp \left(\mathrm{c}_{\delta / 2} \operatorname{deg}(f) s\right)
\end{aligned}
$$

which leads to the contradiction that $\eta \leq 0$. Thus, $m(J(f)) \geq s$, and then $m(I(f)) \geq s$ as well, since $J(f) \subseteq I(f)$.

Let

$$
K \stackrel{\text { def }}{=}(\mathbb{T} \backslash A) \cap I(f) .
$$

Since $m(I(f)) \geq s$ and $m(\mathbb{T} \backslash A)=2 \pi-m(A)$, we have $2 m(K) \geq s+2 \pi-m(A)-$ $2 \pi=(\delta / 2) m(A) /(2 \pi-\delta)$ (cf. 52). ${ }^{11}$ Therefore,

$$
\begin{aligned}
\int_{A} \chi^{p}(f) & \leq \int_{A}\|\chi(f)\|_{L^{\infty}(\mathbb{T})}^{p}=m(A)\|\chi(f)\|_{\infty}^{p} \\
& \leq \frac{4(2 \pi-\delta)}{\delta} m(K)\|\chi(f)\|_{L^{\infty}(\mathbb{T})}^{p}=\frac{4(2 \pi-\delta)}{\delta} \int_{K}\|\chi(f)\|_{L^{\infty}(\mathbb{T})}^{p} .
\end{aligned}
$$

Hence, since $K \subseteq I(f)$,

$$
\int_{A} \chi^{p}(f) \leq \frac{4(2 \pi-\delta)}{\delta} \exp \left(\mathrm{c}_{\delta / 2} \frac{2 \pi-\frac{\delta}{2}}{2 \pi-\delta} p \operatorname{deg}(f) m(A)\right) \int_{K} \chi(f),
$$

\footnotetext{
${ }^{11}$ If $S_{1}$ and $S_{2}$ are two sets then $S_{1}=\left(S_{1} \cap S_{2}\right) \cup\left(S_{1} \backslash S_{2}\right)$ and $S_{2}=\left(S_{1} \cap S_{2}\right) \cup\left(S_{2} \backslash S_{1}\right)$. Hence, $m\left(S_{1}\right)+m\left(S_{2}\right)=2 m\left(S_{1} \cap S_{2}\right)+m\left(S_{1} \backslash S_{2}\right)+m\left(S_{2} \backslash S_{1}\right)$, so that $2 m\left(S_{1} \cap S_{2}\right) \geq$ $m\left(S_{1}\right)+m\left(S_{2}\right)-m\left(S_{1} \cup S_{2}\right)$.
} 
and, finally, since $K \subseteq \mathbb{T} \backslash A$,

$$
\int_{A} \chi^{p}(f) \leq \frac{4(2 \pi-\delta)}{\delta} \exp \left(\mathrm{c}_{\delta / 2} \frac{2 \pi-\frac{\delta}{2}}{2 \pi-\delta} p \operatorname{deg}(f) m(A)\right) \int_{\mathbb{T} \backslash A} \chi(f) .
$$

Theorem 16 is completely proved.

Proof of Theorem 18. First assume that $p=1$. Fix one of the component intervals in $\Delta=\bigcup \Delta_{k}$, say, $\Delta_{k_{0}}$. Then

$$
f(x) u(x) \leq \int_{\Delta_{k_{0}}}\left|(f u)^{\prime}\right|, \quad x \in \Delta_{k_{0}},
$$

so that

$$
\int_{\Delta_{k_{0}}} f u \leq\left|\Delta_{k_{0}}\right| \int_{\Delta_{k_{0}}}\left|(f u)^{\prime}\right|
$$

that is,

$$
\int_{\Delta_{k_{0}}} f u \leq\left|\Delta_{k_{0}}\right| \int_{\Delta_{k_{0}}}\left|f^{\prime}\right| u+\left|\Delta_{k_{0}}\right| \int_{\Delta_{k_{0}}} f\left|u^{\prime}\right|
$$

where the second term on the right-hand side vanishes if $\Gamma=0$. Hence, for $\Gamma>0$,

$$
\int_{\Delta_{k_{0}}} f u \leq\left|\Delta_{k_{0}}\right| \int_{\Delta_{k_{0}}}\left|f^{\prime}\right| u+\left|\Delta_{k_{0}}\right| \sup _{t \in \Delta_{k_{0}}} \frac{\left|u^{\prime}(t)\right|}{u(t)} \int_{\Delta_{k_{0}}} f u .
$$

If $u$ is given by

$$
u(z)=\omega \prod_{j}\left|\sin \frac{z-z_{j}}{2}\right|^{r_{j}}, \quad \omega>0, z_{j} \in \mathbb{C}, z \in \mathbb{C}, r_{j}>0,2 \Gamma \stackrel{\text { def }}{=} \sum_{j} r_{j},
$$

then, by (18),

$$
\left|\Delta_{k_{0}}\right| \sup _{t \in \Delta_{k_{0}}} \frac{\left|u^{\prime}(t)\right|}{u(t)} \leq\left|\Delta_{k_{0}}\right| \frac{\sum_{j} r_{j}}{2} \sup _{\substack{t \in \Delta_{z_{j}} \\ z_{j}}}\left|\cot \left(t-z_{j}\right)\right| \leq \frac{1}{2} .
$$

Therefore, for all $\Gamma \geq 0$,

$$
\int_{\Delta_{k_{0}}} f u \leq 2\left|\Delta_{k_{0}}\right| \int_{\Delta_{k_{0}}}\left|f^{\prime}\right| u .
$$

Since this is true for each component interval of $\Delta$, we obtain

$$
\int_{\Delta} f u \leq 2 \max _{k}\left|\Delta_{k}\right| \int_{\Delta}\left|f^{\prime}\right| u \leq 2 \max _{k}\left|\Delta_{k}\right| \int_{\mathbb{T}}\left|f^{\prime}\right| u
$$

and then (19) follows immediately from Theorem 16 applied with $p=1$ and $\chi \equiv 1$.

Once (19) holds with $p=1$, we can use it with $f$ replaced by $f^{p} \in \operatorname{NGTP}_{p N}$, and then (19) follows for all $1<p<\infty$ from Hölder's inequality.

Proof of Corollary 19.

Step 1. If $\ell=1$ and $j=0$ in (20), then we can apply Theorem 18 after making the following two observations. First, if $t_{n} \in \mathcal{T}^{r}$ then $f \stackrel{\text { def }}{=}\left|t_{n}\right| \in \operatorname{NGTP}_{\operatorname{deg}\left(t_{n}\right)}$ and $\left|f^{\prime}\right|=\left|\left(\left|t_{n}\right|\right)^{\prime}\right| \equiv\left|t_{n}^{\prime}\right|$ everywhere except at the zeros of $f$ (cf. Remark 13). Second, we can choose a constant $c_{1} \in \mathbb{R}^{+}$such that, if for each $n \in \mathbb{N}$ we pick only those intervals $\tau_{i_{k}, n}$ for which $\operatorname{dist}\left(\tau_{i_{k}, n},\{\right.$ zeros of $\left.u\}\right)>\mathrm{c}_{1} / n$, then $\Delta_{k} \stackrel{\text { def }}{=} \tau_{i_{k}, n}$ 
satisfy (18), and $2 \pi-c_{2} / n<m\left(\bigcup \Delta_{k}\right) \leq 2 \pi$ with an appropriate constant $\mathrm{c}_{2} \in \mathbb{R}^{+}$ independently of $n$.

Step 2. If $\ell>1$ and $j=\ell-1$ in (20), then we can apply Step 1 with $t_{n}^{(\ell-1)}$ in place of $t_{n}$ after observing that if the closure of each $\tau_{i n}$ contains at least one zero of $t_{n}$, then, by Rolle's Theorem, the closure of each $\bigcup_{i^{*}=i}^{i+2 \ell-2} \tau_{i^{*}, n}$ contains at least one zero of $t_{n}^{(\ell-1)}$, so that a new set of intervals $\left\{\tau_{i n}^{*}\right\}$ can be formed by joining sufficiently many adjacent ones (or their closures) from the original set $\left\{\tau_{i n}\right\}$ in such a way that for the new set (i) $\tau_{i n}^{*} \subset \mathbb{T}$ are disjoint intervals, (ii) the closure of each $\tau_{i n}^{*}$ contains at least one zero of $t_{n}^{(\ell-1)}$, and (iii) $m\left(\tau_{i n}\right) \leq \mathrm{d}^{*} / n$ with an appropriate constant $\mathrm{d}^{*} \in \mathbb{R}^{+}$.

Step 3. If $\ell>1$ and $0 \leq j<\ell-1$ in (20) then we just write the fraction in (20) as the product of ratios with consecutive derivatives, and use Step 2 with each term.

Proof of Corollary 20. If $j=\ell-1$ in (20), then it follows immediately from Corollary 19 applied with $t_{n}(\cdot) \stackrel{\text { def }}{=} p_{n}\left(\frac{b-a}{2} \cos (\cdot)+\frac{b+a}{2}\right)$. Otherwise, we just write the fraction in (21) as the product of ratios with consecutive derivatives, and apply what we have proved already to each term.

The following lemma is very simple and very useful.

Lemma 28. Let $\Delta=\bigcup_{k} \Delta_{k}$, where $\Delta_{k}$ are disjoint intervals either in $\mathbb{R}$ or in $\mathbb{T}$. Let $1 \leq p<\infty$. Let $f$ be differentiable almost everywhere in each $\Delta_{k}$, and let $|f|^{p}$ be absolutely continuous in each $\Delta_{k}$. Let the closure of each $\Delta_{k}$ contain at least one zero of $f$. Let $u \geq 0$ be Lebesgue measurable in $\Delta$. Then

$$
\|f\|_{L_{u}^{p}(\Delta)} \leq p \sup _{k} m\left(\Delta_{k}\right) \sup _{k}\left(\|u\|_{L^{\infty}\left(\Delta_{k}\right)}\left\|u^{-1}\right\|_{L^{\infty}\left(\Delta_{k}\right)}\right)\left\|f^{\prime}\right\|_{L_{u}^{p}(\Delta)} .
$$

Proof of Lemma 28. Fix one of the component intervals in $\Delta=\bigcup \Delta_{k}$, say, $\Delta_{k_{0}}$. Then

$$
|f(x)|^{p} \leq p \int_{\Delta_{k_{0}}}\left|f^{\prime}\right||f|^{p-1}, \quad x \in \Delta_{k_{0}}
$$

so that

$$
\int_{\Delta_{k_{0}}}|f|^{p} u \leq p m\left(\Delta_{k_{0}}\right)\|u\|_{L^{\infty}\left(\Delta_{k_{0}}\right)}\left\|u^{-1}\right\|_{L^{\infty}\left(\Delta_{k_{0}}\right)} \int_{\Delta_{k_{0}}}\left|f^{\prime} \| f\right|^{p-1} u .
$$

Now add up these inequalities, and then use Hölder's inequality to obtain (53).

Proof of Theorem 21. Inequality (23) follows directly from Lemma 28 by observing that

$$
\frac{u(y)}{u(x)}=\exp \left(\frac{1}{2} \sum_{j} \pm \Gamma_{j} \int_{x}^{y} \cot \frac{z-z_{j}}{2} d z\right) \leq \exp \left(|x-y| \Gamma \pi / \operatorname{dist}\left(\tau_{i_{k}},\left\{z_{j}\right\}\right)\right)
$$

for $x, y \in \tau_{i_{k}}$.

Proof of Corollary 22. If $\ell=1$ and $j=0$ in (24), then it follows directly from Theorem 21. If $\ell>1$ and $j=\ell-1$ in (24), then we can use Rolle's Theorem just as in Step 2 in the proof of Corollary 19. If $\ell>1$ and $0 \leq j<\ell-1$ in (24), then we can proceed again as in Step 3 in the proof of Corollary 19. 
Proof of Corollary 23. We just repeat the proof of Corollary 20. That is, if $j=$ $\ell-1$ in (26) then it follows immediately from Corollary 22 applied with $t_{n}(\cdot) \stackrel{\text { def }}{=}$ $p_{n}\left(\frac{b-a}{2} \cos (\cdot)+\frac{b+a}{2}\right)$, and, otherwise, we write the fraction in (26) as the product of ratios with consecutive derivatives, and apply what we have proved already to each term.

\section{REFERENCES}

1. J. G. Clunie and A. B. J. Kuijlaars, Approximation by polynomials with restricted zeros, J. Approx. Theory 79 (1994), 109-124. MR 95k:30008

2. Z. Ditzian and V. Totik, Moduli of Smoothness, Springer Series in Computational Mathematics, Vol. 9, Springer-Verlag, New York-Berlin, 1987. MR 89h:41002

3. T. Erdélyi, Remez-type inequalities and their applications, J. Comput. Appl. Math. 47 (1993), 167-209. MR 94m:26003

4. T. Erdélyi, Remez-type inequalities on the size of generalized polynomials, J. Lond. Math. Soc. 45 (1992), 255-264. MR 93e:41002

5. T. Erdélyi, A. Máté, and P. Nevai, Inequalities for generalized non-negative polynomials, Constr. Approx. 8 (1992), 241-255. MR 93e:41020

6. P. Erdős, On extremal properties of the derivatives of polynomials, Annals Math. 41 (1940), 310-313. MR 1:323g

7. J. Eröd, On lower bounds of the maximums of certain polynomials, Mat. Fiz. Lapok 46 (1939), 58-83 (Hungarian).

8. G. Freud, Orthogonal Polynomials, Pergamon Press, Oxford, 1971. MR 58:1932 (German original)

9. B. Ja. Levin, On the convergence of sequences of $H$-polynomials, Mat. Sb. 66(108) (1965), 384-397 (Russian). MR 30:4910

10. L. M. Milne-Thomson, The Calculus of Finte Differences, Second (unaltered) edition, Chelsea Publ. Co., New York, 1981. MR 13:245c (1st ed., second printing)

11. I. P. Natanson, Constructive Function Theory, Vol. 1, Uniform Approximation, Frederick Ungar Publ. Co., New York, New York, 1964. MR 33:4529a

12. P. Nevai, Orthogonal Polynomials, Memoirs of Amer. Math. Soc., vol. 213, Providence, Rhode Island, 1979. MR 80k: 42025

13. P. Nevai, Bernstein's inequality in $L^{p}$ for $0<p<1$, J. Approx. Theory 27 (1979), 239-243.

14. F. Riesz and B. Sz.-Nagy, Functional Analysis, translated from the second French edition by L. F. Boron, Ungar, New York, 1955. MR 17:175i

15. I. Schur, Über das Maximum des absoluten Bertrages eines Polynoms in einem gegebenen Intervale, Math. Z. 4 (1919), 271-287.

16. P. Turán, Über die Ableitung von Polynomen, Compositio Math. 7 (1939), 89-95. MR 1:37b

17. J. L. Walsh, Interpolation and Approximation by Rational Functions in the Complex Domain, Colloquium Publications, Vol. 20, Fifth Edition, Amer. Math. Soc., Providence, Rhode Island, 1969. MR 36:1672b (4th ed.)

18. S. P. Zhou, Some remarks on Turán's inequality III: The completion, Anal. Math. 21 (1995), 313-318. MR 96h:41014

Department of Mathematics, Texas A\&M University, College Station, Texas 778433368

E-mail address: terdelyi@math.tamu.edu

$U R L:$ http://www.math.tamu.edu/ ${ }^{\sim}$ terdelyi

Department of Mathematics, The Ohio State University, 231 West 18th Avenue, Columbus, Ohiо 43210-1174

E-mail address: nevai@math.ohio-state.edu

URL: http://www.math.ohio-state.edu/ nevai 